\title{
INFLUÊNCIA DA LARGURA EFETIVA NO DIMENSIONAMENTO DE PONTES E VIADUTOS MISTOS DE AÇO E CONCRETO EM SEÇÃO CAIXÃO
}

\author{
R. S. NICOLETTI ${ }^{1}$, A. S. C. DE SOUZA ${ }^{2}$, A. ROSSI ${ }^{3}$, C. H. MARTINS ${ }^{4}$ \\ Universidade Federal de São Carlos \\ renato_nicoletti@hotmail.com ${ }^{1}$
}

Submetido 04/02/2020 - Aceito 24/04/2020

DOI: $10.15628 /$ holos.2020.9495

\section{RESUMO}

As pontes e viadutos mistos de aço e concreto, em especial na configuração em seção caixão, possuem diversas vantagens estruturais e construtivas. Todavia, há uma grande discrepância nas recomendações para o cálculo da largura efetiva da laje. Por esse motivo, estudase a influência de tal variação no projeto de pontes e viadutos mistos de aço e concreto em seção caixão. Para tanto, foram dimensionados 11 modelos, variando a seção transversal e o vão, nos quais, considerando as recomendações das normas NBR 8800 (2008), AASHTO (2017), EN 1994-2-2 (2005) e as da literatura de Zhu et al. (2015) e Yuan et al. (2016), calculou-se a capacidade resistente ao momento fletor da seção mista e o carregamento distribuído limite para o dimensionamento no Estado Limite de Serviço. Nas análises, comparando os
\end{abstract}

valores de capacidade resistente ao momento fletor médio da NBR 8800 (2008) com a AASHTO (2017), a primeira apresenta valores $17,07 \%$ superiores, em média. Além disso, a NBR 8800 (2008) e o EN 1994-2-2 (2005) permitem carregamentos distribuídos limites superiores, em média, $14,51 \%$ e 13,18\%, respectivamente, aos possibilitados pela AASHTO (2017). Além disso, quanto maior o vão das pontes e viadutos, maior é a variação global na resistência da seção mista. Portanto, a discrepância entre as recomendações normativas exerce influência de forma significativa no processo de dimensionamento, sendo importante a realização de estudos nessa área a fim de uniformizar os procedimentos para determinar a largura efetiva.

PALAVRAS-CHAVE: Largura efetiva, pontes e viadutos mistos de aço e concreto, seção caixão.

\section{INFLUENCE OF EFFECTIVE WIDTH IN THE DESIGN OF COMPOSITE STEEL AND CONCRETE BRIDGES AND VIADUCTS IN BOX SECTION}

\begin{abstract}
Steel and concrete composite bridges and viaducts, especially in the box section, have several advantages. However, there is big discrepancy in the recommendations for calculating the effective width of the slab. For this reason, the influence of such variation on the design of compósite steel and concrete bridges and viaducts in a box section is studied. For that, 11 models were designed, varying the cross section and span, in which, considering the recommendations of the NBR 8800 (2008), AASHTO (2017), EN 1994-2-2 (2005) and those of the literature of Zhu et al. (2015) and Yuan et al. (2016), it was calculated the capacity resistant to the bending moment of the composite section and the limit distributed load for dimensioning in the Service Limit
\end{abstract}

State. In the analysis, comparing the values of capacity resistant to the mean bending moment of NBR 8800 (2008) with AASHTO (2017), the first presents values $17.07 \%$ higher. In addition, NBR 8800 (2008) and EN 1994-2-2 (2005) allow shipments distributed to upper limits, on average, $14.51 \%$ and $13.18 \%$, respectively, to those made possible by AASHTO (2017). Moreover, as the span of bridges and viaducts increases, the greater the overall variation in the resistance of the composite section. Therefore, the discrepancy between the normative recommendations has a significant influence on the design process, and it is important to carry out studies in this area in order to standardize the procedures to determine the effective width.

KEYWORDS: Effective width, bridges and viaducts composite of steel and concrete, box section. 


\section{INTRODUÇÃO}

Por definição, um elemento misto de aço e concreto consiste na associação mecânica, por atrito ou por simples aderência e repartição de cargas, entre um perfil de aço e o concreto, resistindo em conjunto aos esforços solicitantes, numa situação em que se busca a predominância de esforços de tração no aço e de compressão no concreto (DE NARDIN, SOUZA e EL DEBS, 2008).

Os elementos estruturais mistos de aço e concreto consistem em uma solução que provê diversos benefícios, entre os quais se destaca a redução das dimensões dos elementos, do peso próprio e do volume da estrutura, do consumo de aço estrutural, dos custos de fundação e a melhoria da precisão dimensional. Diversos autores têm defendido suas vantagens e realizado estudos sobre seu comportamento nos últimos anos (KOTINDA, 2006; VERÍSSIMO, 2007; DE NARDIN, SOUZA e EL DEBS, 2008; CALDAS, 2008; DE NARDIN e SOUZA, 2008; CHAVES e MALITE, 2011; LIU, BRADFORD e ATAEI, 2017).

Nas vigas mistas de aço e concreto, as tensões na laje não são uniformes, de forma que apresentam um pico de tensões junto à viga, produzindo uma seção não plana após o carregamento. Tal fenômeno é denominado de efeito shear lag. Para considerar este efeito, há o conceito de largura efetiva, realizando uma redução da largura da laje e permitindo que as hipóteses de seção plana sejam mantidas dentro de limites aceitáveis para rotinas de projeto. A Figura 1 ilustra o conceito de largura efetiva.

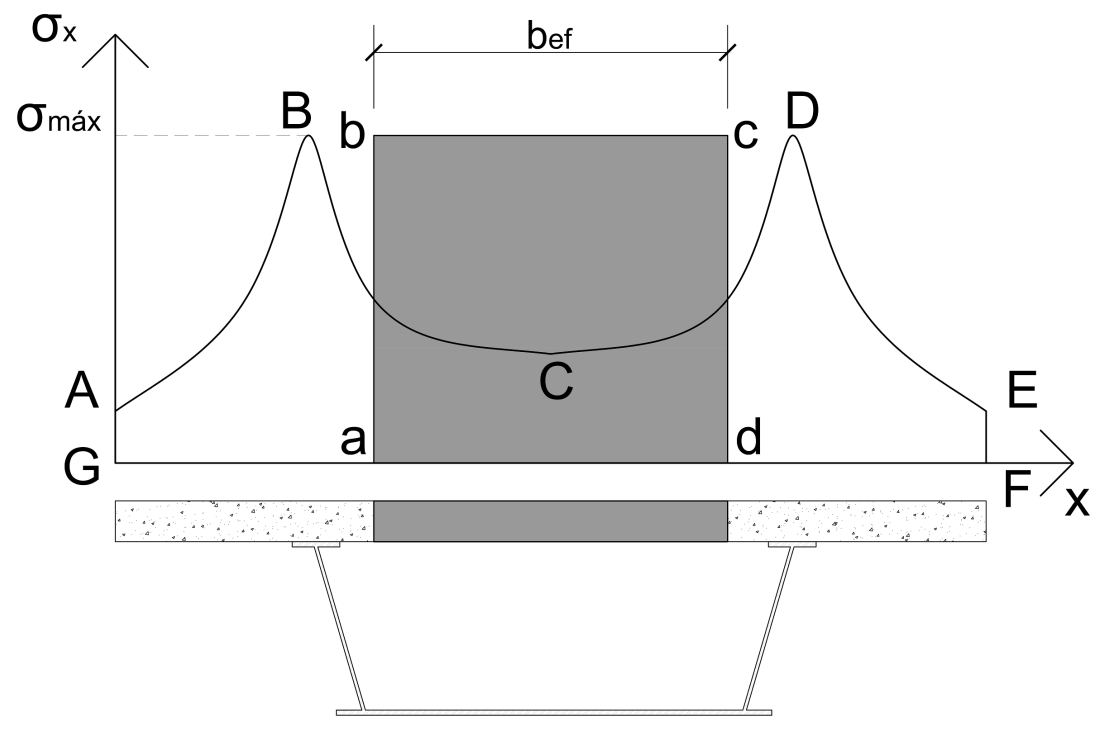

Figura 1: Conceito de largura efetiva.

Fonte: Elaborado pelos autores.

Em que

- $b_{\text {ef }}$ : largura efetiva;

- $\sigma_{x}$ : tensões normais ao longo do comprimento ${ }_{x}$ seção transversal;

- $\sigma_{\text {máx }}$ : tensão normal máxima. 
Portanto, nota-se que apenas uma parcela da laje é considerada efetiva na contribuição da capacidade resistente dos elementos mistos. Na Figura 1, a largura efetiva $\left(b_{e f}\right)$ é determinada quando se iguala a área de distribuição de tensões $A B C D E F G$ à área $a b c d$, delimitando uma largura na qual as tensões são máximas e podem ser consideradas uniformes.

Vários autores têm pesquisado sobre o efeito shear lag e desenvolvido equações para a determinação da largura efetiva de lajes em vigas mistas. Na literatura nacional, dentre os trabalhos que tratam sobre largura efetiva em vigas mistas de aço e concreto, destacam-se o de Reginato, Tamayo e Morsch (2018) e Silva e Dias (2018). Por sua vez, a gama de trabalhos estrangeiros é mais vasta, sobressaindo-se os de Amadio e Fragiacomo (2002), Castro, Elghazouli e Izzuddin (2007), Salama e Nassif (2011), Zhu et al. (2015), Chen e Zhang (2005), Yuan et al. (2016) e Abbu, Ekmekyapar e Oakça (2014). Destes, vale salientar que Zhu et al. (2015) e Yuan et al. (2016) trabalharam especificamente para pontes e viadutos mistos de aço e concreto, tendo proposto expressões de cálculo para a largura efetiva.

Nas normas técnicas, tampouco na literatura, não há nenhuma recomendação para o cálculo da largura efetiva em pontes e viadutos mistos de aço e concreto em seção caixão e, como alternativa, adotam-se as recomendações existentes para vigas de alma cheia, sendo as principais a da norma americana AASHTO (2017) e da europeia EN 1994-2-2 (2005).

Todavia, há uma considerável divergência entre tais recomendações, podendo resultar em soluções mais custosas ou, até mesmo, inseguras para o uso. Visto isso, o presente trabalho possui o objetivo de investigar a influência da largura efetiva no dimensionamento de pontes e viadutos mistos de aço e concreto em seção caixão, levando em conta a faixa de variação da largura efetiva existente entre as recomendações normativas e as da literatura.

\section{REVISÃO BIBLIOGRÁFICA}

Com relação à normatização, para vigas mistas de aço e concreto em alma cheia, principalmente aquelas destinadas a edifícios, a largura efetiva da laje já está estabelecida. Na literatura nacional, a recomendação fica por conta da ABNT NBR 8800 (2008), que trata de estruturas de aço e mistas de aço e concreto. Por sua vez, para pontes e viadutos em vigas mistas de aço e concreto, o cálculo da largura efetiva da laje já está estabelecido em normas, porém, isso não ocorre para vigas mistas em seção caixão. Nesse contexto, as principais normas vigentes são a americana AASHTO (2017) e a europeia EN 1994-2-2 (2005), contudo, as recomendações são para vigas com seção em alma cheia (tipo "I").

Além disso, conforme mencionado anteriormente, as principais recomendações existentes na literatura para cálculo da largura efetiva em pontes e viadutos mistos de aço e concreto são as desenvolvidas por Yuan et al. (2016) e Zhu et al. (2015). Por essa razão, este capítulo aborda tais recomendações juntamente com as das normas citadas. 


\subsection{ABNT NBR 8800 (2008)}

A ABNT NBR 8800 (2008), intitulada "Projeto de estruturas de aço e de estruturas mistas de aço e concreto de edifícios", estabelece que a largura efetiva em vigas biapoiadas de edifícios deve ser menor que ou igual a:

- $1 / 4$ do vão da viga mista;

- metade da distância entre o centro da viga analisada e a viga adjacente;

- distância da linha de cento da viga à borda de uma laje em balanço.

\subsection{AASHTO (2017)}

A AASHTO (2017) define, em seu tópico 6.10.1.1.1e, para pontes e viadutos mistos de aço e concreto em viga "I", que a largura efetiva de cálculo da laje não deverá exceder:

- $1 / 4$ do comprimento do vão da viga;

- a distância de centro a centro de vigas;

- 12 vezes a menor espessura da laje.

Já para vigas que possuem tabuleiro apenas de um lado, a largura efetiva não deverá exceder:

- $1 / 2$ do comprimento do vão da viga;

- metade da distância de centro a centro de vigas;

- 6 vezes a menor espessura da laje.

Convém frisar que o tópico 6.11 da AASHTO (2017) trata sobre vigas mistas de aço e concreto em seção caixão, porém não há recomendações para o cálculo da largura efetiva para esse tipo de seção.

\subsection{EN 1994-2-2 (2005)}

A EN 1994-2-2 (2005) estabelece, em seu tópico 6.1.2, que a largura efetiva para verificação da seção transversal no Estado Limite Último (E.L.S.) deve ser determinada por meio das recomendações explicitadas no seu tópico 5.4.1.2. Nesse item, a largura efetiva é considerada variada ao longo do comprimento do vão da viga. No meio do vão ou em um apoio interno, recomenda-se que a largura efetiva ( $b_{e f}$ ) seja calculada por meio da Equação (1).

$$
b_{e f}=b_{0}+\sum b_{e i}
$$

Em que $b_{0}$ é a distância entre os centros de conectores de cisalhamento; e $b_{e i}$ são os valores das larguras efetivas do tabuleiro para cada um dos lados do centro da viga, admitido como $L_{e} / 8$, em que $L_{e}$ é um vão equivalente que corresponde à distância aproximada entre dois pontos de momento nulo. Estabelece-se, ainda, que $b_{e i}$ não seja superior à largura geométrica $b_{i}$ (Figura 2). Salienta-se, ademais, que $b_{e i}$ trata-se da distância entre o conector de cisalhamento e o ponto 
central entre duas vigas adjacentes, exceto numa extremidade livre, na qual $b_{e i}$ consiste na distância entre o conector de cisalhamento e o bordo da laje.

A largura efetiva nos apoios de extremidade, por sua vez, é calculada por meio da Equação (2).

$$
b_{e f}=b_{0}+\sum \beta_{i} b_{e i}
$$

Nota-se que a única diferença é a existência de um coeficiente redutor $\beta_{i}$, calculado por meio da Equação (3).

$$
\beta_{i}=\left(0,55+\frac{0,025 L_{e}}{b_{e i}}\right) \leq 1,0
$$

A Figura 2 esquematiza as recomendações da EN 1994-2-2 (2005) para o cálculo do comprimento equivalente $L_{e}$.
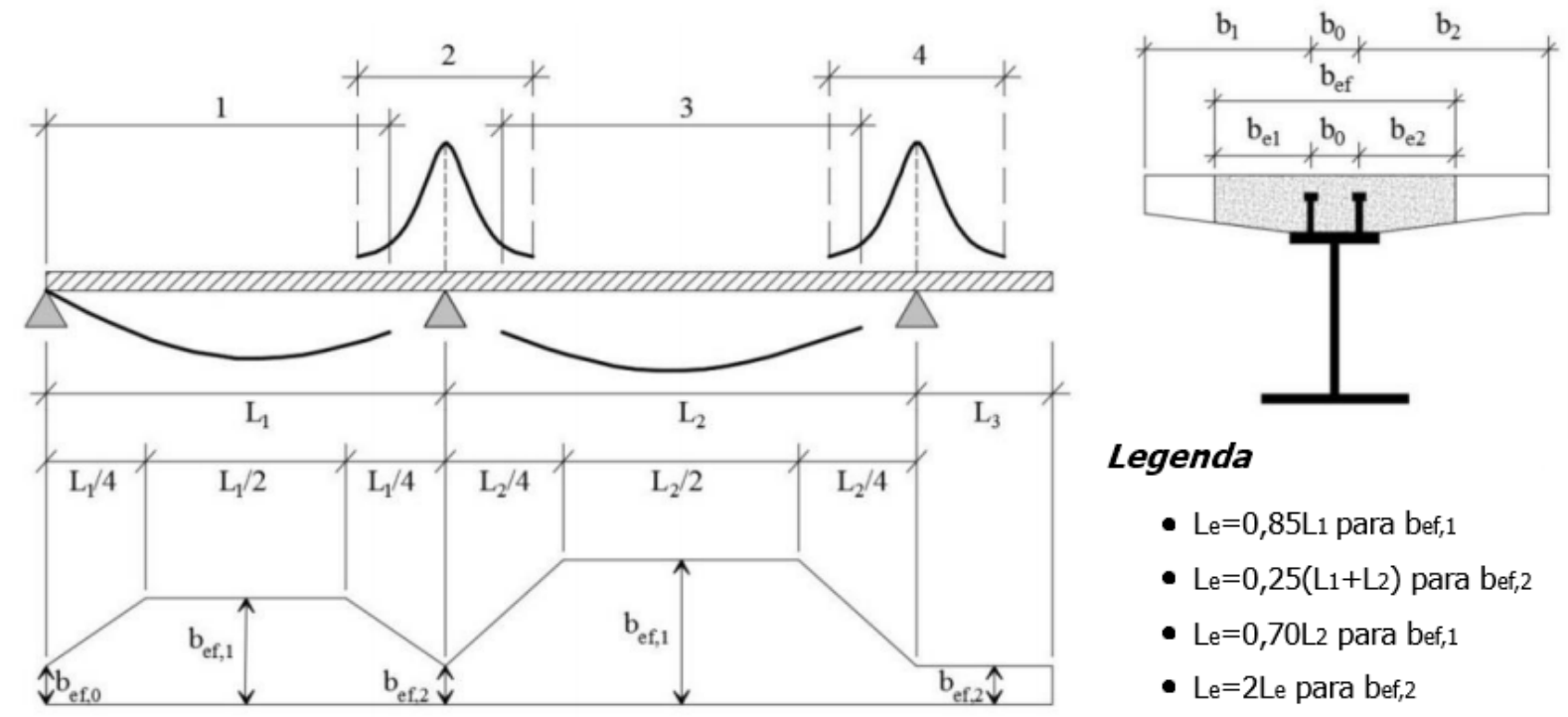

- $\mathrm{Le}=0,85 \mathrm{~L} 1$ para bef, 1

- $\mathrm{Le}=0,25(\mathrm{~L} 1+\mathrm{L} 2)$ para bef,2

- Le=0,70L2 para bef,1

- Le=2Le para bef,2

Figura 2: Cálculo do vão equivalente para determinar a largura efetiva.

Fonte: Adaptado de EN 1994-2-2 (2005).

\subsection{Método de Yuan et al. (2016)}

Yuan et al. (2016) realizaram estudos paramétricos em vigas mistas de aço e concreto, por meio de simulações numéricas fundamentadas no método dos elementos finitos, a fim de analisar quais parâmetros exerciam maior influência na variação da largura efetiva e, com base em seus resultados, propuseram expressões de projeto para o seu cálculo. As principais incógnitas do método estão apresentadas na Figura 3. 


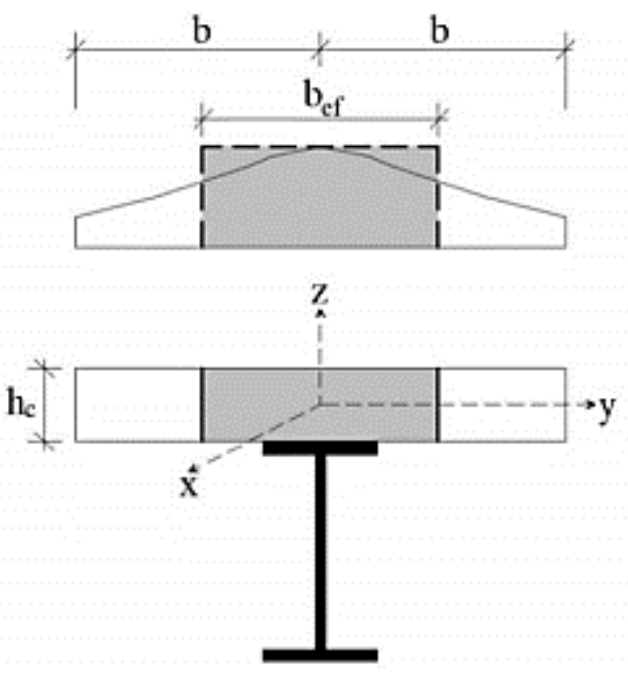

Figura 3: Incógnitas do método de Yuan et al. (2016) para o cálculo da largura efetiva. Fonte: Yuan et al. (2016).

Em que

- $b$ : largura geométrica da laje de concreto medida do centro da viga até a extremidade ou metade da distância de centro a centro de vigas;

- $h_{c}$ : altura da laje de concreto;

- $b_{\text {ef: }}$ largura efetiva.

Com base em seus estudos, Yuan et al. (2016) propuseram a Equação (4) para o cálculo da largura efetiva.

$$
b_{e f}=\eta_{1} \cdot b
$$

O coeficiente $\eta_{1}$ é chamado pelo autor de "coeficiente da largura efetiva". Ele é calculado por meio de outras duas variáveis adimensionais, $x_{1}$ e $x_{2}$, determinadas pelas Equações (5) e (6), respectivamente.

$$
\begin{aligned}
& x_{1}=\frac{L}{b} \\
& x_{2}=\frac{h_{c}}{b}
\end{aligned}
$$

Em que $b$ é a largura da laje de concreto medida do centro da viga até a extremidade ou metade da distância de centro a centro de vigas; $L$ é o comprimento do vão da viga mista; e $h_{c}$ é a altura da laje de concreto.

Finalmente, o coeficiente da largura efetiva é calculado pela Equação (7). 


$$
\eta_{1}=\min \left\{\begin{array}{l}
1-e^{-0,65 x_{1}} \\
\frac{2,83 \cdot 10^{-4}+x_{2}^{2}}{0,001+x_{2}^{2}}
\end{array}\right.
$$

\subsection{Método de Zhu et al. (2015)}

Zhu et al. (2015) executaram ensaios estáticos em pontes mistas de aço e concreto em seções compostas por dois perfis "I" e por uma seção caixão simples, sujeitas a cargas verticais de flexão e cargas de compressão por meio de protensão. Na sequência, diversas análises numéricas foram calibradas com os ensaios realizados e o efeito shear lag foi estudado. Como resultado, foram formuladas algumas equações para o cálculo da largura efetiva.

O método desenvolvido por Zhu et al. (2015) considera o tipo de carregamento para determinar a largura efetiva, bem como a variabilidade dela ao longo do vão. $O$ método é dividido em dois critérios: para cargas de flexão verticais e para cargas axiais de compressão. Este último não será abordado neste trabalho, pois é destinado às vigas mistas de aço e concreto com protensão externa.

Em ambos os métodos, o efeito shear lag é aplicado utilizando um coeficiente adimensional de largura efetiva $(\lambda)$, calculado conforme a Equação (8).

$$
\lambda=\frac{b_{e f}}{b}
$$

A Figura 4 exibe os parâmetros geométricos que são as variáveis do método desenvolvido por Zhu et al. (2015).

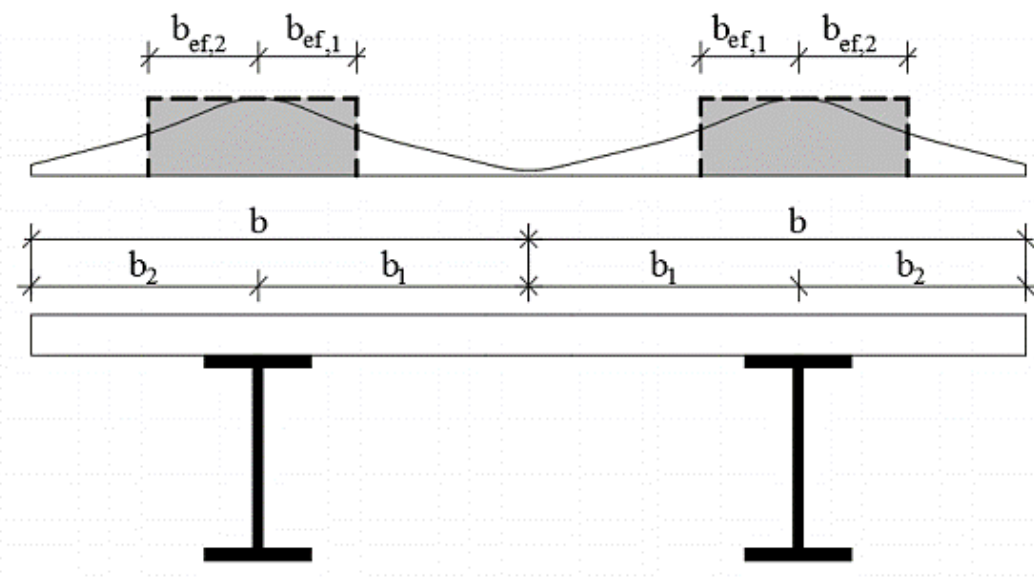

Figura 4: Variáveis do método de Zhu et al. (2015).

Fonte: Reginato (2018), fundamentado em Zhu et al. (2015). 
Em que

- $\quad b$ : largura geométrica da laje de concreto medida do centro da viga até a extremidade ou metade da distância de centro a centro de vigas;

- $b_{1}$ : largura geométrica da laje de concreto medida do centro da viga até o ponto central entre duas vigas;

- $\quad b_{2}$ : largura geométrica da laje de concreto medida do centro da viga até a borda da laje;

- $b_{\text {ef }, 1}$ : largura efetiva da largura geométrica $b_{1}$;

- $b_{e f, 2}:$ largura efetiva da largura geométrica $b_{2}$.

O método para cargas de flexão verticais segmenta o comprimento do vão da viga mista de aço e concreto em pontos de inflexão, de forma a se obter diversos vãos equivalentes. Mais especificamente, considera-se a região dos apoios como sendo aquela que vai da extremidade até $1 / 3$ do comprimento do vão equivalente $\left(I_{e}\right)$ em ambos os lados. Dessa forma, a distribuição da largura efetiva é calculada por meio de um padrão linear que considera três larguras efetivas representativas:

1. a largura efetiva no meio do vão para carregamentos uniformemente distribuídos $\left(b_{e f, u}\right)$, associada ao coeficiente $\lambda_{u}$;

2. a largura efetiva no meio do vão para carregamentos concentrados $\left(b_{e f, c}\right)$, associada ao coeficiente $\lambda_{c}$;

3. a largura efetiva na região dos apoios $\left(b_{e f, s}\right)$, associada ao coeficiente $\lambda_{s}$.

Além disso, consideram-se dois casos de carregamento:

1. sem cargas concentradas, no qual o coeficiente da largura efetiva é igual a $\lambda_{s}$ na região dos apoios e $\lambda_{u}$ na região central. Nos demais pontos, a largura efetiva é uma interpolação linear entre ambos, Figura 5-(a);

2. com cargas concentradas, no qual o vão equivalente é dividido inicialmente em duas partes no ponto de aplicação da carga pontual. Nesse caso, o coeficiente da largura efetiva varia linearmente entre as partes. Ele é $\lambda_{s}$ na região dos apoios, varia linearmente da extremidade até atingir $\lambda_{u}$ em $\frac{2 L_{e}}{3}$ e, em seguida, decresce linearmente até atingir $\lambda_{c}$ no ponto de aplicação da carga. Na porção à direita do ponto de aplicação da carga, o comportamento é simétrico. Caso haja mais de uma força concentrada aplicada, consideram-se novos vãos equivalentes. A Figura 5-(b) ilustra essa situação. 


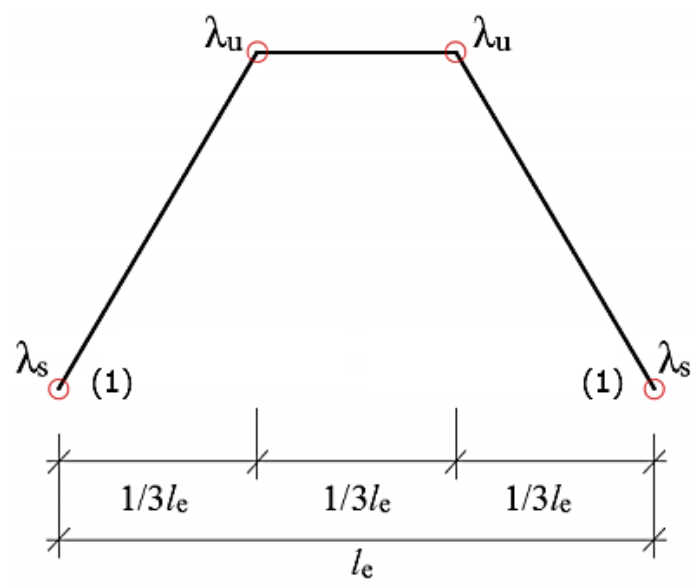

(a)

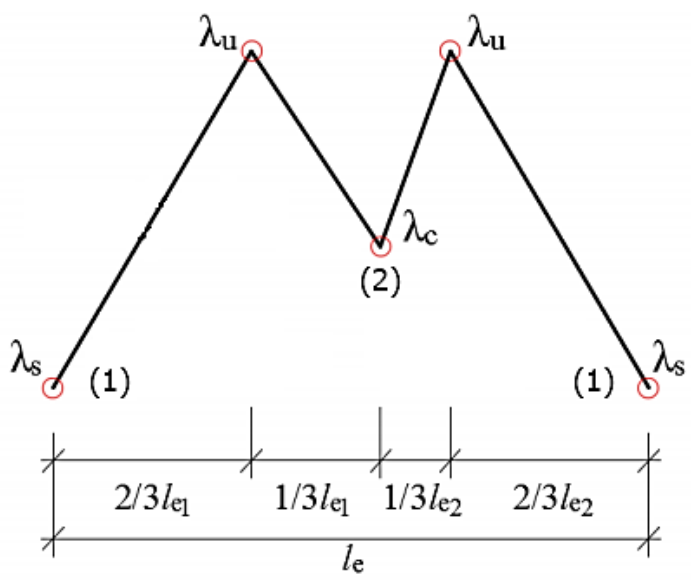

(b)

(1) Ponto de inflexão ou região do apoio

(2) Região de apoio ou ponto de aplicação de carga pontual externa

Figura 5: Padrões de distribuição do coeficiente de largura efetiva do método de Zhu et al. (2015). (a) Caso em que não há cargas concentradas. (b) Caso em que há cargas concentradas.

Fonte: Adaptado de Zhu et al. (2015).

Em que

- $\lambda_{s}$ : coeficiente da largura efetiva nos apoios;

- $\lambda_{u}$ : coeficiente da largura efetiva no meio do vão para carregamentos uniformemente distribuídos;

- $\lambda_{c}$ : coeficiente da largura efetiva no meio do vão para carregamentos pontuais.

- $\left.\right|_{e}$ : comprimento do vão equivalente.

Empregam-se as Equações (9), (10) e (11), respectivamente, para o cálculo dos coeficientes de largura efetiva nos casos de: cargas distribuídas no meio do vão equivalente; carga concentrada no meio do vão equivalente; e na região dos apoios em ambos os casos.

$$
\begin{aligned}
& \lambda_{u}=\frac{b_{i, e f, u}}{b_{i}}=1-e^{-0,212 \cdot\left(\frac{b_{i}}{L}\right)^{-0,1284}} \quad(i=1,2,3) \cdot \\
& \lambda_{c}=\frac{b_{i, e f, c}}{b_{i}}=\frac{1}{3,294 \cdot\left(\frac{b_{i}}{l}\right)^{2}+2,487 \cdot\left(\frac{b_{i}}{L}\right)^{2}+1} \quad(i=1,2,3) \\
& \lambda_{s}=\frac{b_{i, e f, s}}{b_{i}}=1-e^{-0,0652 \cdot\left(\frac{b_{i}}{L}\right)^{-0,313}} \quad(i=1,2,3)
\end{aligned}
$$

\subsection{Análise crítica dos métodos}

Dos métodos apresentados, os prescritos pela AASHTO (2017), por Yuan et al. (2016) e Zhu et al. (2015) são os que consideram a altura da laje, o comprimento do vão e o espaçamento entre 
vigas na seção transversal para determinar a largura efetiva. Por sua vez, a ABNT NBR 8800 (2008) e o EN 1994-2-2 levam em conta apenas o vão e o posicionamento das vigas na seção transversal.

Entretanto, vale ressaltar que os métodos de Yuan et al. (2016) e Zhu et al. (2015) apresentam pouca sensibilidade à variação dos parâmetros analisados.

\section{METODOLOGIA}

Com o intuito de estudar a influência da variabilidade da largura efetiva no dimensionamento de pontes e viadutos mistos de aço e concreto em seção caixão, foram prédimensionados 11 modelos de pontes/viadutos em duplo caixão. Nestes, foram variados o vão, a altura da laje e as dimensões da seção transversal das vigas caixão. A Figura 6 apresenta as simbologias dos parâmetros geométricos das pontes.

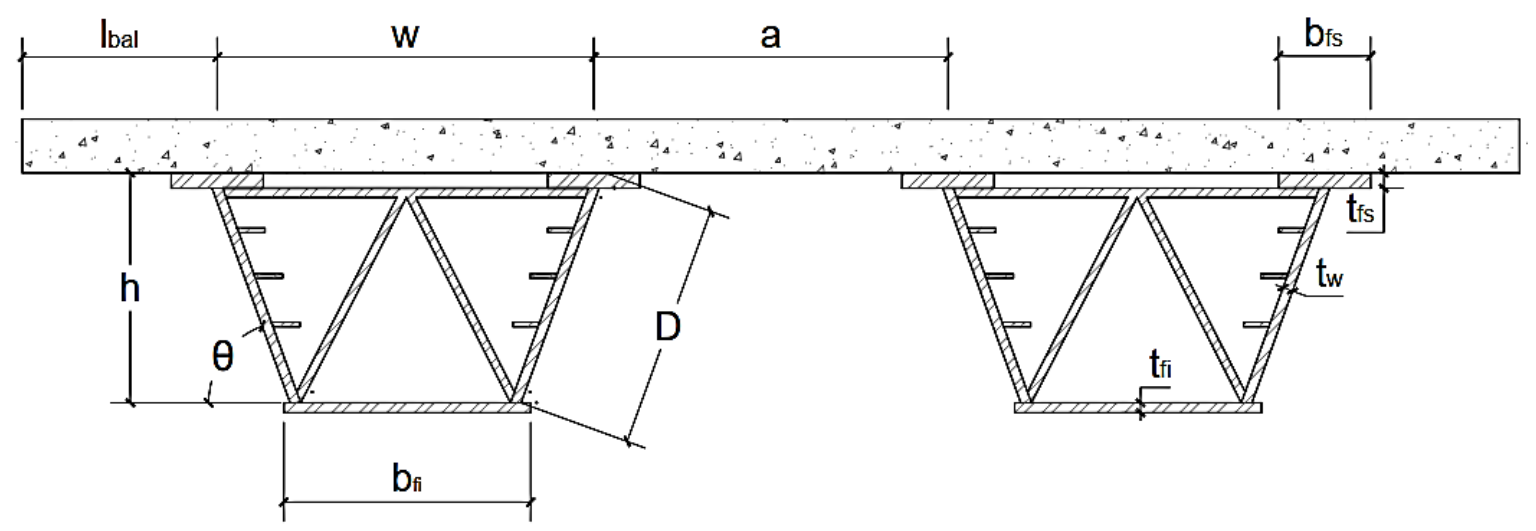

Figura 6: Parâmetros geométricos da seção transversal dos modelos.

Fonte: Elaborado pelos autores.

Em que

- $I_{\text {bal }}$ : comprimento do balanço da laje na seção transversal;

- $\quad w$ : distância entre almas na região superior do caixão;

- a: distância entre almas de caixões paralelos;

- $\quad b_{f s}$ : largura da mesa superior do caixão;

- $\quad b_{f i}$ : largura da mesa inferior do caixão;

- $\quad \theta$ : inclinação da alma do caixão em relação a um plano horizontal;

- $h$ : altura do caixão;

- $D$ : comprimento da alma do caixão;

- $t_{f s}$ : espessura da mesa superior do caixão;

- $t_{f i}$ : espessura da mesa inferior do caixão;

- $t_{w}$ : espessura da alma do caixão.

Em todos os modelos, adotou-se a largura da seção transversal do tabuleiro de $12.800 \mathrm{~mm}$, a largura da mesa superior do caixão de $b_{f s}=350 \mathrm{~mm}$, a largura da mesa inferior de $b_{f i}=2.500 \mathrm{~mm}$ e as espessuras dos perfis foram $t_{f s}=t_{f i}=t_{w}=31,75 \mathrm{~mm}$. Tais valores estão em conformidade com 
o pré-dimensionamento recomendado pela AASHTO (2017). A Tabela 1 apresenta a geometria dos modelos.

Tabela 1: Parâmetros geométricos da seção transversal dos modelos.

\begin{tabular}{c|c|c|c|c|c|c}
\hline Modelo & Vão $[\mathrm{mm}]$ & hlaje $[\mathrm{mm}]$ & hviga $[\mathrm{mm}]$ & $\mathbf{w}[\mathrm{mm}]$ & lbal $[\mathrm{mm}]$ & $\mathbf{a}[\mathrm{mm}]$ \\
\hline 1 & $30.000,00$ & 200,00 & $1.000,00$ & $2.920,00$ & $1.460,00$ & $4.040,00$ \\
2 & $33.000,00$ & 210,00 & $1.100,00$ & $2.960,00$ & $1.480,00$ & $3.920,00$ \\
3 & $36.000,00$ & 220,00 & $1.200,00$ & $3.000,00$ & $1.500,00$ & $3.800,00$ \\
4 & $39.000,00$ & 230,00 & $1.300,00$ & $3.040,00$ & $1.520,00$ & $3.680,00$ \\
5 & $42.000,00$ & 240,00 & $1.400,00$ & $3.080,00$ & $1.540,00$ & $3.560,00$ \\
6 & $45.000,00$ & 250,00 & $1.500,00$ & $3.120,00$ & $1.560,00$ & $3.440,00$ \\
7 & $48.000,00$ & 260,00 & $1.600,00$ & $3.165,00$ & $1.582,50$ & $3.305,00$ \\
8 & $51.000,00$ & 270,00 & $1.700,00$ & $3.210,00$ & $1.605,00$ & $3.170,00$ \\
9 & $54.000,00$ & 280,00 & $1.800,00$ & $3.250,00$ & $1.625,00$ & $3.050,00$ \\
10 & $57.000,00$ & 290,00 & $1.900,00$ & $3.300,00$ & $1.650,00$ & $2.900,00$ \\
11 & $60.000,00$ & 300,00 & $2.000,00$ & $3.330,00$ & $1.665,00$ & $2.810,00$ \\
\hline
\end{tabular}

Fonte: Elaborado pelos autores.

Quanto às propriedades dos materiais, utilizou-se para a laje um concreto com módulo de elasticidade longitudinal de $30.589 \mathrm{MPa}$ e resistência característica à compressão de $30 \mathrm{MPa}$. Para as vigas metálicas, foi utilizado o aço ASTM A242, com módulo de elasticidade longitudinal de 200.000 MPa e resistência característica ao escoamento de $345 \mathrm{MPa}$. Além disso, considerou-se interação total entre os materiais em todos os modelos.

A fim de investigar a influência da variação da largura efetiva no dimensionamento no Estado Limite Último, calculou-se o momento fletor resistente $\left(M_{R d}\right)$ da seção mista, considerando os diversos métodos para o cálculo da largura efetiva expostos na seção 2 . Em razão da grande rigidez dos caixões metálicos, a linha neutra plástica (LNP) da seção mista situou-se sobre eles em todos os modelos. Desse modo, para o cálculo da capacidade resistente ao momento fletor da seção mista plastificada, empregaram-se as equações (12), (13), (14) e (15), cujos parâmetros estão expostos na Figura 7.

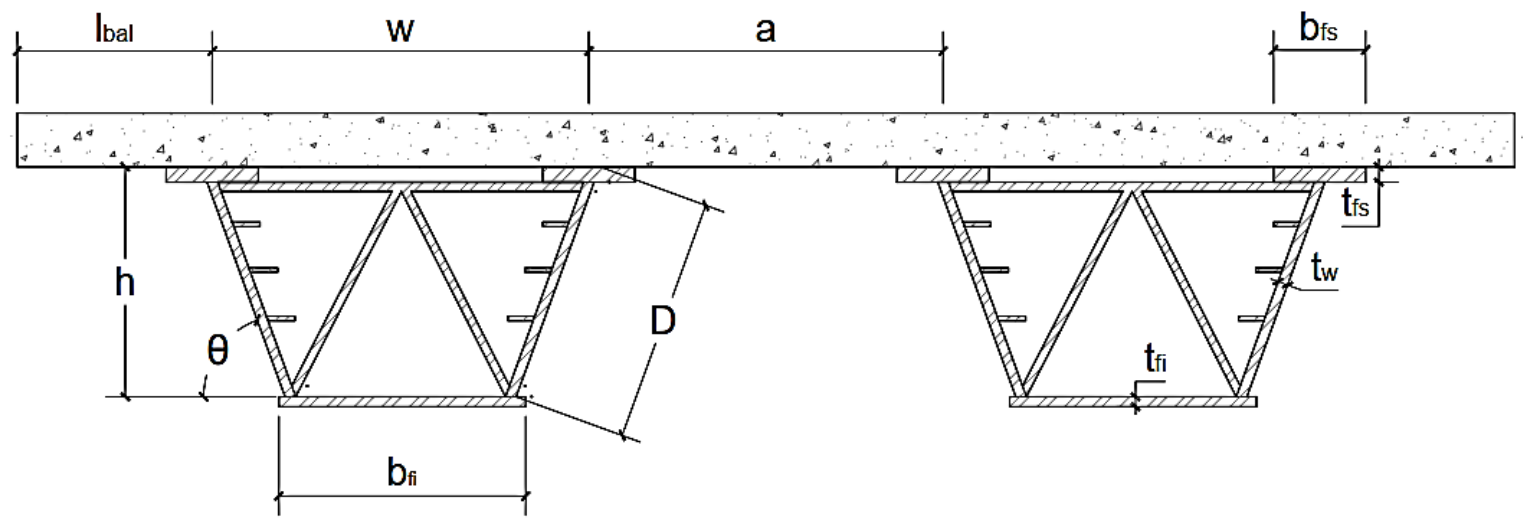

Figura 7: Parâmetros para o cálculo da capacidade resistente ao momento fletor - LNP no caixão metálico. Fonte: Elaborado pelos autores. 


$$
\begin{aligned}
& C_{\text {Aço }}=A_{s}^{\prime} \cdot f_{y d} \\
& T_{\text {Aço }}=A_{s} \cdot f_{y d} \\
& C_{\text {Concreto }}=0,85 \cdot f_{c d} \cdot b_{e f} \cdot h_{\text {laje }} \\
& M_{R d}=F_{\text {Tração }} \cdot z=F_{\text {Compressão }} \cdot z
\end{aligned}
$$

Em que

- $C_{\text {Concreto }}$ força de compressão na laje;

- $\quad C_{A c ̧ o}$ : força de compressão na viga caixão;

- $T_{\text {Aço }}$ : força de tração na viga caixão metálica;

- $\quad$ : distância entre os pontos de aplicação da resultante de tração e de compressão;

- $f_{c d}$ : resistência de cálculo do concreto à compressão;

- $\quad h_{\text {laje }}:$ altura da laje;

- $A_{s}$ : área de aço do caixão abaixo da LNP;

- $\quad A_{s}^{\prime}$ : área de aço do caixão acima da LNP;

- $f_{y d}$ : resistência de cálculo do aço à tração;

- $\quad b_{e f}$ : largura efetiva da laje.

Por sua vez, a fim de avaliar como a faixa de variação da largura efetiva existente na literatura e nas normativas afeta o dimensionamento no Estado Limite de Serviço (E.L.S.), considerou-se a deflexão limite de $L$ / 800 para cada modelo. Esta limitação é fixada pela norma brasileira de pontes e viadutos mistos de aço e concreto, ABNT NBR 16694 (2018) - que se encontra em fase de consulta pública - e pela AASHTO (2017). Feito isso, utilizando-se a Equação (16), determinou-se qual o carregamento distribuído $(q)$ que ocasiona tais flechas para cada um dos métodos de determinação da largura efetiva.

$$
\delta=\frac{L}{800}=\frac{5 q L^{4}}{384 E I_{t r}} \rightarrow q=\frac{384 E I_{t r}}{4000 L^{3}}
$$

Em que

- $\quad$ : comprimento do vão;

- $\quad E$ : módulo de elasticidade longitudinal do aço; 
- $I_{t r}$ : inércia calculada no centro geométrico da seção homogeneizada totalmente em aço.

\section{RESULTADOS E DISCUSSÕES}

A Tabela 2 apresenta os valores da largura efetiva dos 11 modelos, calculados de acordo com as recomendações da ABNT NBR 8800 (2008), AASHTO (2017), EN 1994-2-2 (2005), Yuan et al. (2016) e Zhu et al. (2015).

Tabela 2: Largura efetiva dos modelos para cada uma das recomendações citadas.

\begin{tabular}{c|c|c|c|c|c}
\hline \multirow{3}{*}{ Modelo } & \multicolumn{5}{|c}{ LARGURA EFETIVA (mm) } \\
\cline { 2 - 6 } & $\begin{array}{c}\text { NBR 8800 } \\
\mathbf{( 2 0 0 8 )}\end{array}$ & $\begin{array}{c}\text { AASHTO } \\
\mathbf{( 2 0 1 7 )}\end{array}$ & $\begin{array}{c}\text { EN 1994 2-2 } \\
\mathbf{( 2 0 0 5 )}\end{array}$ & $\begin{array}{c}\text { Yuan et al. } \\
\mathbf{( 2 0 1 6 )}\end{array}$ & $\begin{array}{c}\text { Zhu et al. } \\
\text { (2015) }\end{array}$ \\
\hline 1 & $3.750,00$ & $2.400,00$ & $3.537,50$ & $5.452,26$ & $1.574,82$ \\
2 & $4.125,00$ & $2.520,00$ & $3.856,25$ & $5.526,41$ & $1.591,74$ \\
3 & $4.500,00$ & $2.640,00$ & $4.175,00$ & $5.592,74$ & $1.607,30$ \\
4 & $4.875,00$ & $2.760,00$ & $4.493,75$ & $5.652,13$ & $1.621,71$ \\
5 & $5.250,00$ & $2.880,00$ & $4.812,50$ & $5.705,37$ & $1.635,13$ \\
6 & $5.625,00$ & $3,000,00$ & $5.131,25$ & $5.753,16$ & $1.647,69$ \\
7 & $6,000,00$ & $3.120,00$ & $5.450,00$ & $5.796,18$ & $1.659,49$ \\
8 & $6.375,00$ & $3.240,00$ & $5.768,75$ & $5.834,80$ & $1.670,60$ \\
9 & $6.300,00$ & $3.360,00$ & $6.087,50$ & $5.869,60$ & $1.681,11$ \\
10 & $6.200,00$ & $3.480,00$ & $6.406,25$ & $5.900,80$ & $1.691,06$ \\
11 & $6.140,00$ & $3.600,00$ & $6.725,00$ & $5.929,43$ & $1.700,54$ \\
\hline
\end{tabular}

Fonte: Elaborado pelos autores.

A Figura 8 exibe a variação da largura efetiva para os modelos analisados.

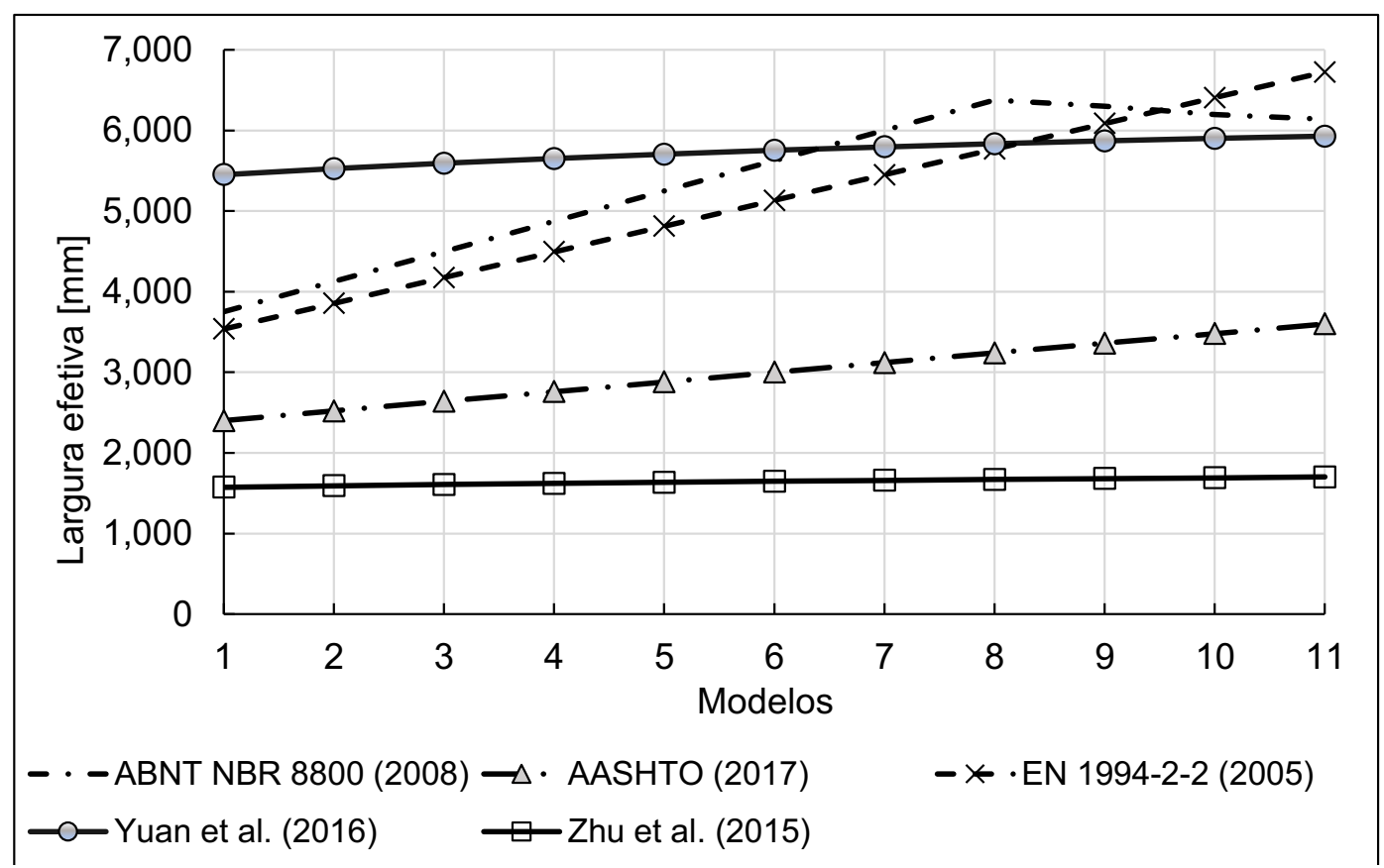

Figura 8: Valores da largura efetiva dos modelos para cada uma das recomendações abordadas.

Fonte: Elaborado pelos autores. 
De imediato, observa-se que há uma grande divergência no valor da largura efetiva nos modelos em função da norma ou recomendação adotada. Mais detalhadamente, tem-se que as recomendações de Zhu et al. (2015) resultam em larguras efetivas bem conservadoras e com pouquíssima variação entre os modelos, pois este método não considera a altura da laje em seus cálculos, e o comprimento do vão exerce pouca influência na determinação de ${ }^{b_{\text {ef }}}$. Em termos de conservadorismo, em seguida, há a largura efetiva calculada de acordo com AASHTO (2017), que foi limitada pela altura da laje em todos os modelos. No mais, as equações da ABNT NBR 8800 (2008), do EN 1994-2-2 (2005) e de Yuan et al. (2016) apresentam os maiores valores de largura efetiva, alternando entre si.

Vale comentar que a largura efetiva calculada com base nos procedimentos de Yuan et al. (2016) também não variou significativamente em função da variação da geometria da seção transversal e do comprimento do vão, mesmo o método considerando tais parâmetros. Observouse que o coeficiente $\eta_{1}$ manteve-se praticamente constante em todos os modelos.

Além disso, as larguras efetivas calculadas de acordo com o EN 1994-2-2 (2005) possuem a maior sensibilidade frente à variação da geometria da seção transversal e do vão dos modelos, exibindo um coeficiente de variação de 30,24\%. Enquanto isso, o método de Zhu et al. provê maior constância nos valores da largura efetiva, com um coeficiente de variação de $19,27 \%$.

A Figura 9 exibe graficamente a influência de tal variação no cálculo do momento fletor resistente da seção mista, principal limitante do dimensionamento no Estado Limite Último.

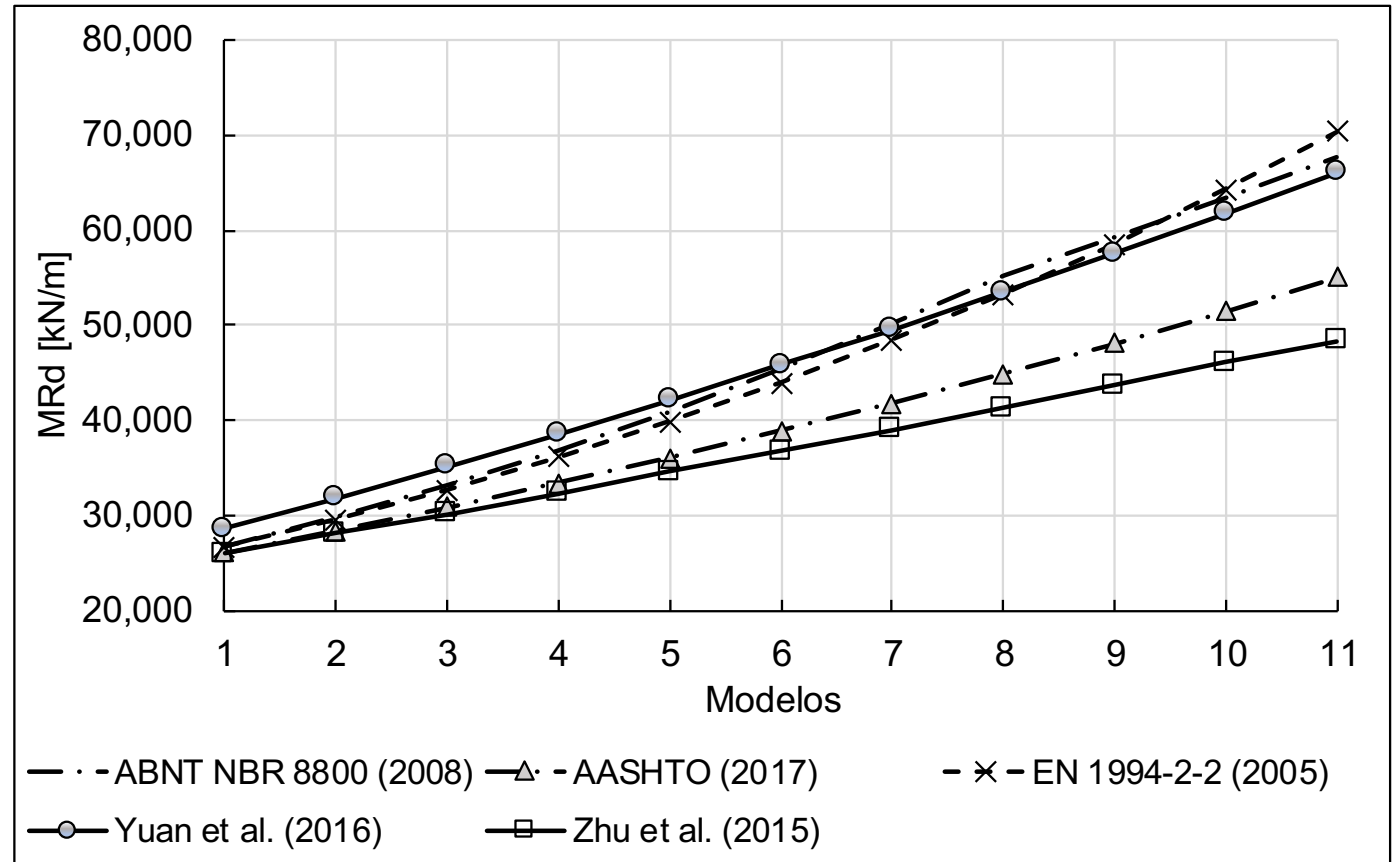

Figura 9: Influência da variação da largura efetiva no momento fletor resistente da seção mista dos modelos. Fonte: Elaborado pelos autores.

As recomendações da ABNT NBR 8800 (2008), do EN 1994-2-2 (2005) e de Yuan et al. (2016) resultaram nos maiores valores de momento fletor resistente da seção mista, com valores médios de $46.256,28 \mathrm{kN} \cdot \mathrm{m}, 45.767,61 \mathrm{kN} \cdot \mathrm{m}$ e $46.402,08 \mathrm{kN} \cdot \mathrm{m}$. Enquanto isso, as equações da AASHTO (2017) e de Zhu et al. (2015) ocasionaram valores médios de MRd de 39.509,86 kN·m e 36.908,15 
$\mathrm{kN} \cdot \mathrm{m}$. Verifica-se ainda que, comparando os valores de capacidade resistente ao momento fletor médio da ABNT NBR 8800 (2008) com os da AASHTO (2017), a primeira apresenta valores 17,07\% superiores, afetando diretamente a viabilidade de um projeto, uma vez que exigirá um maior consumo de aço e concreto para produzir seções com maior rigidez.

Em razão da grande variação da largura efetiva ocasionada pelos procedimentos da norma europeia, verifica-se que ela influencia significativamente nos limitantes do dimensionamento no E.L.U quando a geometria da seção transversal e o comprimento do vão variam.

Por fim, considerando a deflexão limite de $L / 800$ para o dimensionamento no Estado Limite de Serviço, a Figura 10 apresenta o carregamento distribuído correspondente que ocasiona tais deflexões, levando em conta a largura efetiva calculada por cada um dos métodos.

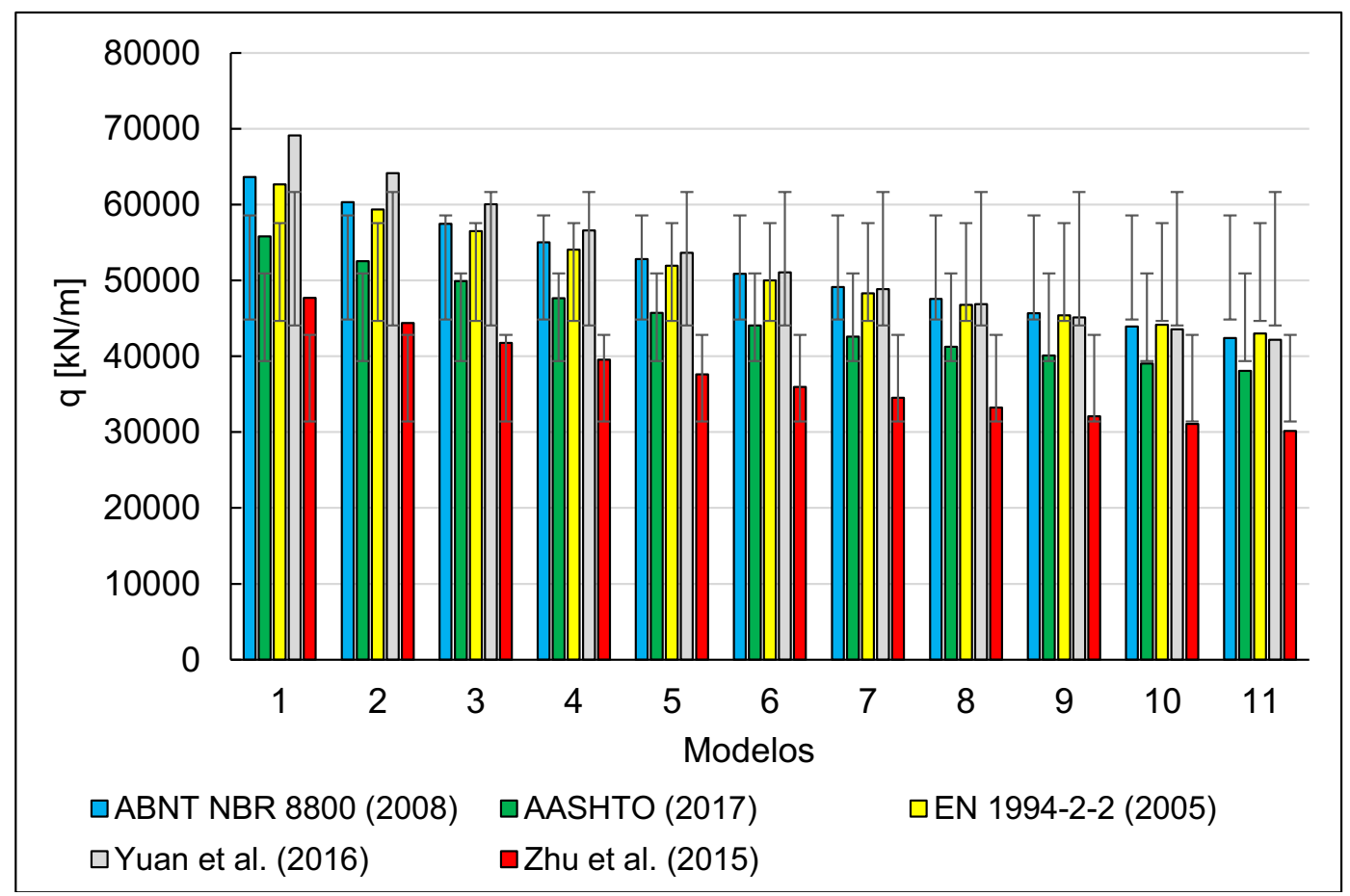

Figura 10: Influência da variação da largura efetiva no carregamento distribuído máximo para atender ao E.L.S. nos modelos.

Fonte: Elaborado pelos autores.

Visando atender ao principal critério do dimensionamento no E.L.S. da ABNT NBR 16694 (2018) e da AASHTO (2017) - principais referências normativas para o dimensionamento de pontes e viadutos mistos de aço e concreto no Brasil - a largura efetiva calculada de acordo com as recomendações da ABNT NBR 8800 (2008), do EN 1994-2-2 (2005) e de Yuan et al. resultaram nas maiores médias de cargas limites distribuídas, sendo de, respectivamente, $51.712,78 \mathrm{kN} / \mathrm{m}$, $51.111,84 \mathrm{kN} / \mathrm{m}$ e $52.842,48 \mathrm{kN} / \mathrm{m}$. Por sua vez, as larguras efetivas mais conservadoras, provenientes dos métodos de cálculo da AASHTO (2017) e de Zhu et al. (2015), acarretaram valores de $q$ médios de, respectivamente, $45.159,61 \mathrm{kN} / \mathrm{m}$ e $37.095,07 \mathrm{kN} / \mathrm{m}$. Assim, verifica-se que a ABNT NBR 8800 e o EN 1994-2-2 permitem cargas distribuídas limites superiores, em média, $14,51 \%$ e $13,18 \%$, respectivamente, às possibilitadas pela AASHTO (2017).

No mais, o valor de $q$ apresentou coeficientes de variação oscilando entre $12,00 \%$ e $15,87 \%$ em todos os métodos, não havendo um destaque significativo para nenhum deles. Isso 
significa que, em praticamente todos os métodos, alterações na geometria e no comprimento do vão provocam mudanças na largura efetiva que afetam o cálculo da deflexão limite em, no máximo, $15,87 \%$.

Notou-se ainda que quanto maior o comprimento do vão, maior a variação global na resistência da seção mista em função do critério adotado. Nos modelos analisados, notou-se uma variação global máxima de $45,40 \%$ no modelo 11 , comparando as recomendações do EN 1994-2-2 com o método de Zhu et al. (2015).

\section{CONCLUSÃO}

A discrepância existente entre as recomendações para o cálculo da largura efetiva de pontes e viadutos mistos de aço e concreto em seção caixão influencia significativamente no processo de dimensionamento. Quando se comparam os métodos, verifica-se um maior conservadorismo por parte da AASHTO (2017) em relação aos demais, exibindo valores de $M_{R d}$ médios 17,07\% inferiores aos da ABNT NBR 8800 e carregamentos limites máximos, em média, $14,51 \%$ inferiores à ABNT NBR 8800 e 13,18\% ao EN 1994-2-2.

A média dos valores de momento fletor resistente calculada pelas recomendações do EN 1994-2-2 (2005) exibiu um coeficiente de variação de 30,24\%, significando que alterações na geometria da seção transversal e do comprimento do vão provocam variações deste percentual na resistência da capacidade resistente ao momento fletor da seção mista. Por sua vez, no E.L.S., o coeficiente de variação dos carregamentos limites oscilou entre $12,00 \%$ e $15,87 \%$ entre os métodos, indicando variações médias nessa faixa quando a geometria da estrutura é modificada.

Ademais, vale frisar que quanto maior o comprimento do vão das pontes e viadutos, maior foi a variação global na resistência da seção mista, isto é, maior foi a oscilação entre o método que apresentou a maior capacidade resistente ao momento fletor e o que apresentou a menor. Para o modelo 11, observou-se uma variação global máxima de 45,40\%, comparando as recomendações do EN 1994-2-2 com o método de Zhu et al. (2015).

Portanto, faz-se importante ressaltar a necessidade de estudos adicionais acerca da largura efetiva em pontes e viadutos mistos de aço e concreto, a fim de uniformizar os procedimentos para a sua determinação, com o objetivo de garantir segurança e economia para tais estruturas.

\section{REFERÊNCIAS}

AMADIO, C.; FRAGIACOMO, M. (2002). Effective width evaluation for steel-concrete composite beams. Journal of Constructional Steel Research, v. 58, n. 3, p. 373-388.

AMERICAN ASSOCIATION OF STATE HIGHWAY OFFICIALS (AASHTO). (2017). LRFD Bridge Design Specifications, 8th Edition, American Association of State Highway and Transportation Officials. Washington, DC.

ASSOCIAÇÃO BRASILEIRA DE NORMAS TÉCNICAS (ABNT). (2018). NBR 16694: Projeto de pontes rodoviárias de aço e mistas de aço e concreto. Rio de Janeiro. 
ASSOCIAÇÃO BRASILEIRA DE NORMAS TÉCNICAS (ABNT). (2008). NBR 8800: projeto de estruturas de aço e de estruturas mistas de aço e concreto de edifícios. Rio de Janeiro.

CALDAS, R. B. (2008). Análise numérica de estruturas de aço, concreto e mistas em situação de incêndio. 2008. Tese (Doutorado em Estruturas) - Escola de Engenharia da Universidade Federal de Minas Gerais, Universidade Federal de Minas Gerais, Belo Horizonte.

CASTRO, J. M.; ELGHAZOULI, A. Y.; IZZUDDIN, B. A. (2007). Assessment of effectivee slab widths in composite beams. Journal of Constructional Steel Research, v. 63, n. 10, p. 1317-1327.

CHAVES, I. A.; MALITE, M. (2011). Viga mista de aço e concreto constituída por perfil formado a frio preenchido. Cadernos de Engenharia de Estruturas, v. 12, n. 56, p. 79-96.

DE NARDIN, S.; SOUZA, A. S. C. (2008). Sistemas de vigas mistas contínuas e semicontínuas para edifícios. In: XXXIII Jornadas Sudamericanas de Ingenieria Estructural, 2008, Santiago. XXXIII Jornadas Sudamericanas de Ingenieria Estructural. Santiago: Universidad Central de Chile. v. 1. p. 1-10.

DE NARDIN, S.; SOUZA, A. S. C.; EL DEBS, A. L. H. C. (2008). Comportamento conjunto em elementos mistos de aço e concreto: Dispositivos Mecânicos. In: Congresso Latino Americano de Construção Metálica, 3, 2008, São Paulo/SP. Anais do Construmetal 2008. São Paulo: ABCEM.

EUROPEAN COMMITTEE FOR STANDARDIZATION (CEN). (2005). Eurocode 4: Design of composite steel and concrete structures - Part 2: General rules and rules for bridges. EN 1994-2-2. Brussels.

KOTINDA T. I. (2006). Modelagem numérica de vigas mistas aço-concreto simplesmente apoiadas: ênfase ao estudo da interface laje-viga. 121p. Dissertação (Mestrado em Engenharia de Estruturas) - Universidade de São Paulo, São Carlos.

LIU, X.; BRADFORD, M. A.; ATAEI, A. (2017). Flexural performance of innovative sustainable composite steel-concrete beams. Engineering Structures, v. 130, p. 282-296.

REGINATO, L. H.; TAMAYO, J. L. P.; MORSCH, I. B. (2018). Finite element study of effective width in steel-concrete composite beams under long-term service loads. Latin American Journal of Solids and Structures, v. 15, n. 8.

SALAMA, T.; NASSIF, H. H. (2011). Effective flange width for composite steel beams. Journal of Engineering Research, v. 8, n. 1, p. 28-43.

SILVA, A. R.; DIAS, L. E. S. (2018). Análise numérica do efeito da interação parcial na avaliação da largura efetiva de vigas mistas. Revista IBRACON de Estruturas e Materiais, v. 11, n. 4, p. 757778.

VERÍSSIMO, G. S. (2007). Desenvolvimento de um conector de cisalhamento em chapa dentada para estruturas mistas de aço e concreto e estudo do seu comportamento. 316p. Tese (Doutorado em Engenharia de Estruturas) - Universidade Federal de Minas Gerais, Belo Horizonte. 
YUAN, H.; DENG, H.; YANG, Y.; WEIJIAN, Y.; ZHENGGENG, Z. (2016). Element-based effective width for deflection calculation of steel-concrete composite beams. Journal of Constructional Steel Research, v. 121, p. 163-172.

ZHU, L.; NIE, J. G.; LI, F. X.; JI, W. Y. (2015). Simplified analysis method accounting for shear-lag effect of steel-concrete composite decks. Journal of Constructional Steel Research, v. 115, p. 62-80. 